Proc. of the 15th Int. Workshop on Slow Positron Beam Techniques and Applications, Prague, September 2-6, 2019

\title{
Simulating Positron to Positronium Conversion in Nanostructured Materials
}

\author{
F. Guatieri ${ }^{a, b, *}$, S. MARiazzi ${ }^{b, c}$ And R.S. Brusa ${ }^{b, c}$ \\ ${ }^{a}$ Forschungs-Neutronenquelle Heinz Maier-Leibnitz (FRM II), Lichtenbergstr. 1, 85748 Garching, Germany \\ ${ }^{b}$ Physics Department, University of Trento, Via Sommarive, 14, 38123 Povo, Trento TN, Italy \\ ${ }^{c}$ TIFPA/INFN, University of Trento, Via Sommarive 14, 38123 Trento TN, Italy

\begin{abstract}
Silicon-based nanochanneled converters for the production of cold positronium (Ps) have been introduced in 2010 and since then provided a reliable source of cold Ps to a variety of experiments. With the goal of advancing the optimization of such converters, we have formulated a classical model to describe the production and cooling of Ps in nanochanneled converters. The simulation of this process poses several challenges due to the complexity of the geometry in which it takes place. We will here briefly give an overview of our model and discuss several simplifications of the simulation process which, without altering significantly the simulation results, reduce the computational costs enough to allow for systematic scans of the nanochanneled converter construction parameter space.
\end{abstract}

DOI: 10.12693/APhysPolA.137.113

PACS/topics: positronium, positron, cooling, thermalisation, silicon, silica, nanostructures, Monte Carlo

\section{Introduction}

A variety of modern scientific endeavors benefit from the availability of an abundant source of slow Ps; their number includes spectroscopy experiments [1,2], gravity experiments using excited Ps [3] or antihydrogen produced by charge exchange [4], and the production of a Bose-Einstein condensate of Ps atoms [5,6].

An abundant source of cold Ps can be provided by silica-coated silicon nanochanneled plates (NCPs). In recent years, NCPs have been the workhorse of spectroscopy experiments on Ps atoms carried out at AEgIS [7-11]. The performance of the NCPs and the characteristics of the Ps cloud they generate had been already characterized experimentally [12-14], but only very recently we were able to provide a quantitative model of the inner working of the NCPs capable of predicting correctly the experimental results in most experimental settings [15-17]. In this contribution, we will provide a quick overview of this model and as we do so we will detail a few of the strategies that have allowed to streamline what would otherwise have been an overwhelmingly demanding simulation.

\section{Geometric model}

We begin formulating our model of NCP converters by providing a geometric description of their nanoscopic structure. We devised a model of a single NCP channel as the union of the volumes of a fixed number of geometric shapes, specifically, truncated cones positioned

\footnotetext{
* corresponding author; e-mail: francesco.guatieri@frm2.tum.de
}

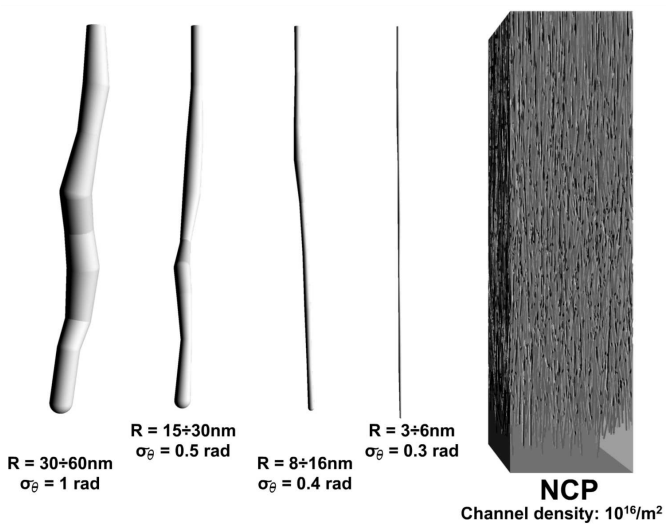

Fig. 1. From the left to the right, four examples of channels generated with our model: the leftmost channel was generated using exaggerated parameters for its radius and its tortuousness, as we progress towards the right the parameters are moved closer to realistic values with the last channel being an example of a realistic channel. The box on the right is a cutout of a NCP similar to those employed in our simulations, rendered with a translucent shader, the channel density is so high that single channels are barely distinguishable. A detailed description of the construction parameters of our geometric model and their function has been given elsewhere $[15,16]$.

end-to-end and joined by spheres. The orientation and size of the constituent geometric elements of each channel are generated procedurally with a pseudo-random number generator. The probability distributions used to draw the size and position of the channel components are parametrized, and the parameters describing them are chosen as to mimic the expected structure of the NCP $[15,17]$. The four leftmost items in Fig. 1 are examples of channels produced by our model with different settings for the generation parameters. 
We have then defined the geometric model of an NCP as an (infinite) half-space, from the surface of which are branching inside a number of channels per unit of surface equal to expected density of channels in a real NCP. In a typical NCP the length of the channels can reach $2 \mu \mathrm{m}$ and their density can exceed $3 \times 10^{16} / \mathrm{m}^{2}$. An adequate description of a single channel requires at least 16 primitives ( 8 spheres and 8 truncated cones). As tens of thousands of channels populate the region of the NCP being traversed by a particle, the number of geometric primitives that are involved in the simulation of the evolution of a single $\mathrm{e}^{+} / \mathrm{Ps}_{\mathrm{s}}$ inside the $\mathrm{NCP}$ can easily reach hundreds of thousands $[15,17]$. The rightmost item of Fig. 1 shows a graphical representation of a small portion of such structure generated with typical parameters. In order to tame the complexity of such geometry, we eschew the use of triangle meshes and instead opted for analytical methods to compute the intersection of $\mathrm{e}^{+} / \mathrm{Ps}$ trajectories with the surface of the channels [17]. Even so, it is still paramount to partition a large NCP in smaller and simpler fragments, pre-compute the bounding boxes of the geometric primitives employed, and organize them in octree structures. This allows to drastically limit the amount of objects to be considered each time that the trajectory of an $\mathrm{e}^{+} / \mathrm{Ps}$ has to be re-computed, which happens after every discrete interaction of the particle with the solid medium.

\section{Implantation}

Employing the technical methods listed in the previous section, it is possible to compute the implantation profiles of $\mathrm{e}^{+}$in NCPs without having to resort to supercomputers [16]. We modeled the interaction between high energy $(>100 \mathrm{eV}) \mathrm{e}^{+}$and silicon based on the Penelope model as included in Geant4. When the $\mathrm{e}^{+}$kinetic energy decreases below $100 \mathrm{eV}$ we switch to an effective interaction up until they reach a kinetic energy of $1 \mathrm{eV}$ and their implantation can be considered concluded.

An important question is whether it is necessary to consider the entirety of the NCP structure to compute the correct implantation profile. Our tests show that this is the case only when the energy of the impinging $\mathrm{e}^{+}$ results in an average implantation depth which is comparable with the diameter of the channels (up to $\approx 10$ times their diameter, that is $\approx 3 \mathrm{keV})[15,16]$. When higher implantation energies are employed it is possible to use less computationally intensive geometries, such as spherical porous structures, to predict to extremely high accuracy the correct implantation profile [16]. In this case the size and density of the pores will have to be chosen as to mimic the fraction of empty space found within the NCP structure. Alternatively, it is possible to employ a solid material filling an half-space and whose density in the interaction model in proximity of the surface has been reduced in proportion to the average void volume present at a given depth. This model is computationally inexpensive but produces a shape of the implantation

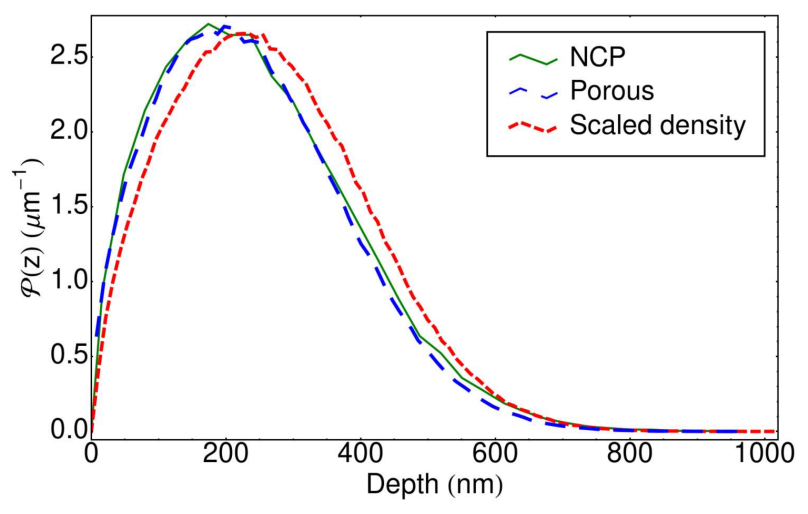

Fig. 2. Implantation profile for a $7 \mathrm{keV} \mathrm{e} \mathrm{e}^{+}$beam impinging on a silicon nanochanneled plate, porous silicon with the same amount of empty space and a model of silicon in which the material density has been reduced proportionally to the amount of empty space in the NCP. It can be seen how the porous material is an excellent substitute for the full NCP model, whereas the reduced density model gives a good, but less precise, approximation of the implantation profile.

profile that is slightly distorted compared to a simulation performed with nanochannels or spherical pores [16]. In Fig. 2 we compare the implantation profile obtained with an $\mathrm{e}^{+}$beam at $7 \mathrm{eV}$ according to three models: the one describing the entire NCP geometry, the one based on the porous model, and the one obtained by rescaling the density of the solid material.

\section{Conversion}

The $\mathrm{e}^{+}$implanted inside the NCP thermalize and diffuse in the bulk of the material until they either annihilate or they reach the silica-coated surface of a nanochannel. In the latter case, circa $40 \%$ of the $\mathrm{e}^{+}$are converted into Ps [17].

The diffusion of the $\mathrm{e}^{+}$can be simulated with the Monte Carlo method as a random walk. In the NCPs employed in our experiments the forest of nanochannels is dense enough to capture quickly all of the $\mathrm{e}^{+}$that diffuse within the thick of it; therefore the depth at which the Ps is generated is, in the region comprised between the surface and the end of the channels, distributed similarly to the implantation profile. The porous geometry mentioned in Sect. 3 is able to correctly reproduce the distribution of the depth at which Ps is generated [17] as long as the implantation energy is, as in Sect. 3, high enough. An even coarser approximation can be obtained by skipping the diffusion process of the $\mathrm{e}^{+}$in the material. In this case, however, the implantation profile has to be truncated at a depth equal to the length of the channels and the contribution provided by the $\mathrm{e}^{+}$implanted more deeply will have to be estimated with a diffusive process [18] and added to the Ps generation profile. In Fig. 3 examples of the profiles that are obtained with the three different approximations are shown. 


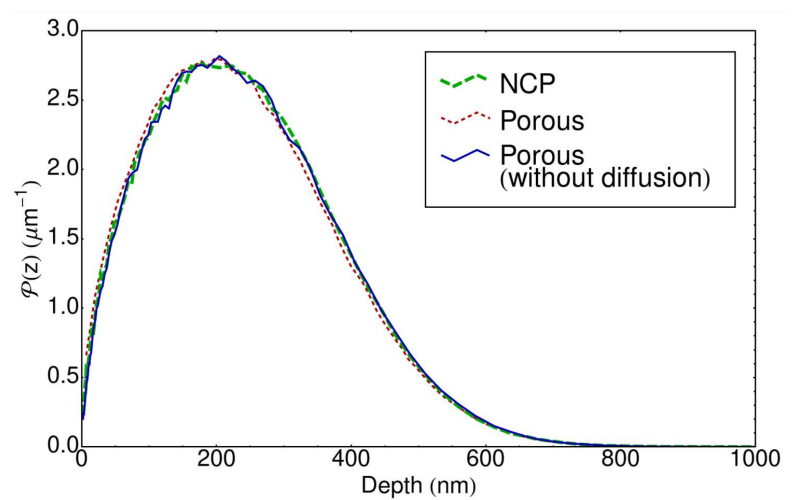

Fig. 3. Depth distribution of the Ps production sites for a $7 \mathrm{keV} \mathrm{e}^{+}$beam impinging on an NCP, its approximation computed on the porous model employed already in Fig. 2 and, for comparison, the implantation depth profile at the same energy. We can see that at the presented implantation energies the three profiles are extremely close to one another. For implantation energies below $3 \mathrm{keV}$ both approximations will start to break down as the exact geometry of the converter begins to play a major role in determining the outcome of the computation. For implantation energies greater than $10 \mathrm{keV}$ the implantation depth profile will show a tail that in the production depth distribution is cut due to the finite length of the channels.

\section{Cooling}

We have formulated a classical model for the transport and cooling of Ps inside of the NCP channels [17], approach that applies to NCPs whose channels are neither extremely cold nor extremely thin [19]. In our model we describe the bouncing of Ps against the walls of the channel as an elastic scattering process having an angular cross-section which is independent of the Ps kinetic energy and an energy exchange derived from a modified version of Sauder's model for Ps cooling in gas moderators $[20,17]$. The fact that the angular cross-section is independent of the Ps energy allows to split the simulation effort in three phases:

1. Firstly, we determine the number of Ps-wall interactions that are needed to exit the nanochannels, assuming an infinitely-lived Ps. Specifically, we compute the discrete probability distribution $\mathcal{S}(n)$ that a Ps atom will escape the nanochannel forest after having interacted exactly $n$ times. This constitutes the most computationally heavy part of the simulation.

2. The second part consists of simulating the evolution $\mathcal{P}_{n}\left(E_{\mathrm{Ps}}\right)$ of the Ps kinetic energy $\mathcal{P}\left(E_{\mathrm{Ps}}\right)$ distribution, as a function of the number of interactions $n$. This results in a series of continuous probability distributions in the Ps atom energy. Since this simulation does not need to make use of the geometric model it is much less computationally intensive than the first.
3. The third step consists of computing the fraction $F_{S}(n)$ of the produced Ps that will survive a given number of interactions with the channel walls without decaying. $F_{S}(n)$ is potentially as costly to compute as $\mathcal{S}(n)$. However, at the expense of a small approximation the computational cost can be made negligible.

\subsection{Computing $\mathcal{S}(n)$}

We compute $\mathcal{S}(n)$ through Monte Carlo simulations in which Ps atoms are generated within the NCP geometry according to the depth distribution previously determined and then their trajectories are propagated within the channel structure. By performing this simulation separately from the simulation of the implantation and diffusion in the bulk, we lose information about the correlation between the propensity of a given nanochannel to capture diffusing $\mathrm{e}^{+}$and the amount of Ps that will start to thermalize from that specific channel. To the best of our abilities, we could not identify any effect in the results of our simulations stemming from the loss of this correlation. On the other hand, there is a clear computational advantage in computing the implantation and diffusion profile of the $\mathrm{e}^{+}$in a simplified model instead of the full NCP.

The cost of computing the trajectory of a Ps atom in an NCP is much greater than that of simulating implantation and diffusion of a single $\mathrm{e}^{+}$as the implantation process requires to simulate order of hundreds discrete interactions, while the propagation of a Ps in a nanochannel can easily take $10^{5} \div 10^{6}$ interactions, each of which requires addressing the complex NCP geometry. We observed that employing a single nanochannel, with a shape that is randomly chosen for each single Ps evolution can result in an $\mathcal{S}(n)$ similar to one given by the full NCP simulation, provided that the radius of the nanochannel is carefully chosen. To do so we proceed as follows. We compute via Monte Carlo simulation the average distance $d_{B}$ that the Ps travels between two successive interactions with the walls of the NCP. We then re-scale the average diameter of the channels used as a substitute for the NCP geometry so that the value of $d_{B}$ in these channels will be equal to the $d_{B}$ computed for the full NCP geometry.

In Fig. 4 we compare the distributions $\mathcal{S}(n)$ computed using the NCP geometry and a single channel with an appropriately adjusted radius.

\subsection{Evolution of the Ps energy distribution}

The simulation of the evolution of the Ps atom energy distribution $\mathcal{P}\left(E_{\mathrm{Ps}}\right)$ as a function of the number of Ps-wall interactions $n$ has a manageable computational cost, nonetheless the output of this simulation being a long series of energy distributions $\mathcal{P}_{n}\left(E_{\mathrm{Ps}}\right)$ can consist of a sizeable amount of data. We can exploit the fact that $\mathcal{P}\left(E_{\mathrm{Ps}}\right)$ evolves quickly as it undergoes the first interactions and very slowly at the end of the thermalisation to 


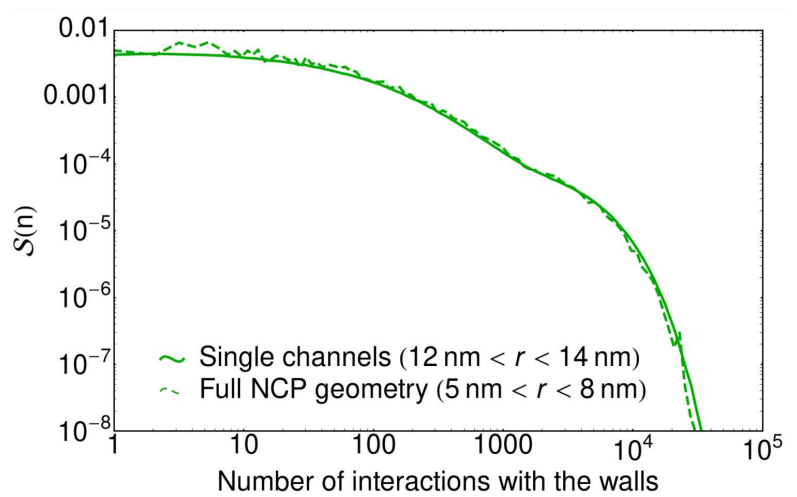

Fig. 4. The discrete distribution $\mathcal{S}(n)$ as computed through the entire NCP geometry or by using single channels whose radius has been adjusted to simulate the parameter $d_{B}$ given by the full NCP geometry; as can be observed, single channels can suitably predict the behavior of the entire NCP. In these simulations an implantation energy of $7 \mathrm{keV}$ was employed, which implants most $\mathrm{e}^{+}$in the thick of the nanochannel forest. The bump appearing above $1 \mathrm{keV}$ is caused by Ps diffusing downwards, hitting the bottom of the channel and then diffusing upwards.

cache these simulations efficiently. We have verified that recording only few stages of the simulation, employing a spacing that increases linearly with $n$, is an effective method of characterizing the entire evolution.

The main parameter that in our model determines $\mathcal{P}_{n}\left(E_{\mathrm{Ps}}\right)$ is the effective mass $M$ that we associate to the wall to model the dynamic of the interaction $[15,17]$. We have also found that it is possible to employ the energy evolution $\mathcal{P}_{n}\left(E_{\mathrm{Ps}}\right)$ computed for a certain effective $M_{1}$ to predict, to a certain degree of approximation, the evolution relative to a different effective mass $M_{2}$ by scaling the subscript $n$ by a factor $M_{1} / M_{2}$.

\subsection{Computing $F_{S}(n)$}

Computing the fraction $F_{S}(n)$ of Ps which has survived after $n$ interactions with the walls would require, in principle, to employ the entire NCP geometry. This is due to the fact that Ps atom decay in the channels takes place according to two different phenomena: either due to pick-off while interacting with the wall, or in-flight due to spontaneous decay in 3 gamma photons. The latter decay probability is proportional to the time of flight $t_{B}$ between successive interactions, which in turn depends on the Ps energy and the distance between interaction sites. In practice, the value of $d_{B}$, computed previously, can be effectively used to approximate $t_{B}$, allowing to express $F_{S}(n)$ in closed form as a function of $F_{S}(n-1)[15,17]$.

\subsection{Dependence on simulation parameters}

We will now briefly illustrate the parametric dependences of $\mathcal{S}(n), \mathcal{P}_{n}\left(E_{\mathrm{Ps}}\right)$, and $F_{S}(n)$ on the simulation parameters.
1. $\mathcal{S}(n)$ depends on the constructive parameters of the NCP (i.e., on its geometry) and on the implantation energy of the $\mathrm{e}^{+}$.

2. $\mathcal{P}_{n}\left(E_{\mathrm{Ps}}\right)$ depends on the temperature of the NCP converter and on the effective mass $M$ employed to model the Ps interaction with the channel walls.

3. $F_{S}(n)$ depends on all the parameters mentioned above, and also on the pick-off probability $p$ associated with each $\mathrm{P}_{\mathrm{S}}$-wall interaction.

Notice how $\mathcal{S}(n)$ and $\mathcal{P}_{n}\left(E_{\mathrm{Ps}}\right)$, which constitute most of the computational cost of the simulation, depend on two disjoint sets of parameters. It is thus possible to compute them for sets of parameters that are deemed to be of interest to then use the results to finalize the simulation for all combinations of the explored parameter ranges. Doing this results in a quadratic improvement in the runtime when scanning large parameter spaces.

\section{Conclusions}

The simulation of an NCP converter is a complex and computationally intensive endeavor. We found several strategies to reduce its complexity and cost, such as using surrogate geometries, ignoring $\mathrm{e}^{+}$diffusion in dense nanostructures, or splitting simulation steps according to their dependence on the simulation parameters. Several of these techniques can be hopefully adapted to be used to simulate $\mathrm{e}^{+} / \mathrm{Ps}$ behavior in nanostructures different from the NCP, thus allowing the theoretical exploration of a wider range of potential $\mathrm{e}^{+}$to Ps converters.

\section{Acknowledgments}

We would like to thank Dr. Davide Orsucci for his help during the redaction of the present proceeding.

\section{References}

[1] D.B. Cassidy, Eur. Phys. J. D 72, 53 (2018).

[2] D.B. Cassidy, P. Crivelli, T.H. Hisakado, L. Liszkay, V.E. Meligne, P. Perez, H.W.K. Tom, A.P. Mills Jr., Phys. Rev. A 81, 012715 (2010).

[3] M. Doser, C. Amsler, A. Belov, et al. (AEgIS collaboration), Class. Quant. Gravit. 29, 18184009 (2012).

[4] A. Kellerbauer, M. Amoretti, A.S. Belov, et al. (AEgIS collaboration), Nucl. Instrum. Methods Phys. Res. B 266, 351 (2008).

[5] D.B. Cassidy, S.D. Hogan, Int. J. Mod. Phys. Conf. Ser. 30, 1460259 (2014).

[6] P. Crivelli, D.A. Coole, S. Friederich, Int. J. Mod. Phys. Conf. Ser. 30, 1460257 (2014).

[7] S. Aghion, C. Amsler, A. Ariga, et al. (AEgIS collaboration), Phys. Rev. A 94, 012507 (2016).

[8] S. Aghion, C. Amsler, M. Antonello, et al. (AEgIS collaboration), Phys. Rev. A 98, 013402 (2018). 
[9] C. Amsler, M. Antonello, A. Belov, et al. (AEgIS collaboration), Phys. Rev. A 99, 033405 (2019).

[10] G. Consolati, S. Aghion, C. Amsler, et al. (AEgIS collaboration), Acta Phys. Pol. A 132, 1443 (2017).

[11] R. Caravita, S. Aghion, C. Amsler, et al. (AEgIS collaboration), Acta Phys. Pol. B 48, 1583 (2017).

[12] S. Mariazzi, P. Bettotti, S. Larcheri, L. Toniutti, R.S. Brusa, Phys. Rev. B 81, 235418 (2010).

[13] S. Mariazzi, P. Bettotti, R.S. Brusa, Phys. Rev. Lett. 104, 243401 (2010)

[14] S. Mariazzi, L. Di Noto, G. Nebbia, R.S. Brusa, J. Phys. Conf. Series 618, 012039 (2015).

[15] F. Guatieri, Ph.D. Thesis, Università degli studi di Trento, 2018.
[16] F. Guatieri, S. Mariazzi, R.S. Brusa, Eur. Phys. J. D 72, 11198 (2018).

[17] F. Guatieri, S. Mariazzi, L. Penasa, G. Nebbia, C. Hugenschmidt, R.S. Brusa, "Modeling of classical Positronium cooling in $\mathrm{Si}$ nanochannels and experimental indication of a transition to quantum behavior at cryogenic temperatures", Phys. Rev. B, to be published.

[18] R. Krause-Rehberg, H.S. Leipner, Positron Annihilation in Semiconductors, Springer, Berlin 1999, p. 52.

[19] S. Mariazzi, A. Salemi, R.S. Brusa, Phys. Rev. B 78, 085428 (2008).

[20] W.C. Sauder, J. Res. Natl. Bur. Stand. 72A, 91 (1968). 